\title{
An Approximate Stance Map of The Spring Mass Hopper with Gravity Correction For Nonsymmetric Locomotions
}

\author{
Ömür Arslan, Uluc̣ Saranlı and Ömer Morgül
}

\begin{abstract}
The Spring-Loaded Inverted Pendulum (SLIP) model has long been established as an effective and accurate descriptive model for running animals of widely differing sizes and morphologies, while also serving as a basis for several hopping robot designs. Further research on this model led to the discovery of several analytic approximations to its normally nonintegrable dynamics. However, these approximations mostly focus on steady-state running with symmetric trajectories due to their linearization of gravitational effects, an assumption that is quickly violated for locomotion on more complex terrain wherein transient, non-symmetric trajectories dominate. In this paper, we introduce a novel gravity correction scheme that extends on one of the more recent analytic approximations to the SLIP dynamics and achieves good accuracy even for highly non-symmetric trajectories. Our approach is based on incorporating the total effect of gravity on the angular momentum throughout a single stance phase and allows us to preserve the analytic simplicity of the approximation to support our longer term research on reactive footstep planning for dynamic legged locomotion. We compare the performance of our method in simulation to two other existing analytic approximations and show that it outperforms them for most physically realistic non-symmetric SLIP trajectories while maintaining the same accuracy for symmetric trajectories.
\end{abstract}

\section{INTRODUCTION}

Effective, programmable control of dynamic legged locomotion is still among difficult unsolved problems faced by the mobile robotics community. Current progress in this area can partly be attributed to the discovery of the SpringLoaded Inverted Pendulum (SLIP) model (illustrated in Fig. $1)$, initially motivated by biomechanical observations $[1,2]$ and later adopted and refined by robotics researchers [14]. Somewhat surprisingly, subsequent research in biomechanics established the SLIP model, despite its apparent simplicity, as a very accurate descriptive model for running animals of widely varying sizes and morphologies $[5,8]$. In parallel, a succession of one-legged hopping robots with SLIP-like morphologies such as Raibert's hoppers [14], the ARLMonopods [11], the Bow-Leg design [18] and the BiMASC leg [13] demonstrated that dynamic locomotion is not only feasible but also has significant energetic and behavioral advantages. These developments led to an increasing belief that the SLIP model may be more than just a descriptive model that fits biological data, but also a control target whose dynamics are an effective and appropriate abstraction for

Ö.Arslan is with the Dept. of Electrical \& Electronics Eng., Bilkent University, 06800 Ankara, Turkey omur@ee.bilkent.edu.tr

U.Saranlı is with the Dept. of Computer Engineering, Bilkent University, 06800 Ankara, Turkey saranli@cs.bilkent.edu.tr

Ö.Morgül is with the Dept. of Electrical \& Electronics Eng., Bilkent University, 06800 Ankara, Turkey morgul@ee.bilkent.edu.tr running behaviors [9]. Evidence to this end was provided by Raibert's robots as well as work on active embedding of SLIP dynamics within more complex morphologies $[3,15]$.

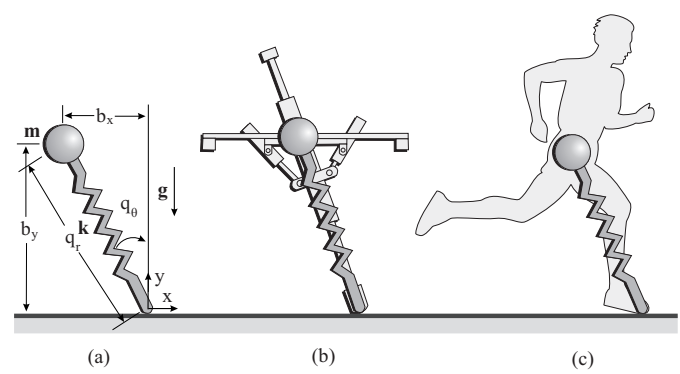

Fig. 1. (a) The SLIP model, (b) Raibert's hopper, (c) A human runner.

This point of view led to the development of control strategies that explicitly operate on the SLIP model itself. Some of these are based on intuitive observations [14], while others seek accurate and preferably analytic approximations to the nonlinear model dynamics $[10,16]$. Such approximations turn out to provide critical tools in the stability analysis of locomotion as well as the design of high performance control and planning algorithms for robot runners while being much more generally applicable than numerical alternatives such as the interpolation based alternatives presented in [7].

Since the stance dynamics of SLIP under the effect of gravity are nonintegrable [12], several approximate alternatives have been proposed in the literature. Most notably, Schwind and Koditschek used a generalization of the meanvalue theorem to obtain an iterative yet analytic approximation to the stance dynamics [17]. Under certain assumptions, the performance of their approximations is shown to increase with each iteration, eventually converging to true SLIP trajectories. Another alternative is presented by Geyer et al. in [10], wherein a much simpler analytic approximation to the spring mass hopper dynamics is derived based on various assumptions specific to the SLIP model. Both methods focus on steps that are symmetric around the vertical, where the effect of gravitational acceleration can be either neglected or linearized with only a minor impact on accuracy.

In reality, however, humans, animals and robots inevitably need to locomote on a variety of irregular terrain (e.g. grass, gravel, rock field, etc.), for which steady-state is never reached and transient, non-symmetric motions dominate. Under these conditions, assumptions based on either linearization of gravity or conservation of angular momentum are no longer applicable, making most of currently available approximations inaccurate. Fig. 2 illustrates the effect of 


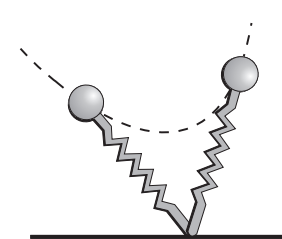

(a)

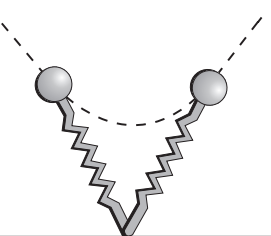

(b)

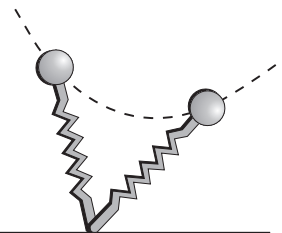

(c)
Fig. 2. The total gravity effect on the angular momentum at the end of the stance phase compared to touchdown instant: (a) decreasing effect on magnitude, (b) the angular momentum is the same since it is a symmetric gait, (c) increasing effect on magnitude.

gravity on the angular momentum of SLIP under different, non-symmetric trajectory conditions.

As noted above, one of our longer term goals is the design of reactive planning controllers for dynamic legged robots. To this end, accurate, preferably analytic characterization of a robot's behavioral repertoire is critical, particularly if overall stability and performance guarantees are required [6]. This necessitates analytic approximations for the SLIP model that perform well even for trajectories on irregular terrain that may never converge to a symmetric steady-state. Such an analytic approximation promises to be extremely valuable for motion planning for the SLIP model and related robots.

In this paper, we propose a novel gravity correction scheme to substantially increase the accuracy of method in [10] for non-symmetric SLIP trajectories and compare our results with approximations presented in both [17] and [10]. The paper continues with the SLIP model and associated terminology, We then summarize previous approximate stance maps and continue in Section III with the proposed gravity correction method. This is followed by the performance analysis and a comparison with existing approximations. Finally, we conclude the paper with a review of our work and a summary of open research topics.

\section{BACKGROUND}

\section{A. The SLIP Model}

The SLIP model consists of a point mass connected to a massless compliant leg as illustrated in Fig. 3(a). During locomotion, the system alternates between stance and flight phases. During stance, the toe remains stationary on the ground with no torque applied to the leg whereas in flight, the body follows a ballistic trajectory. Transition to and from the stance phase occurs with the touchdown and liftoff events, respectively. Two important events are the apex, where the body height is at its maximum and the bottom, where leg compression is maximal. These events and associated subphases are all illustrated in Fig. 3(b). Furthermore, Table I details the notation we use throughout the paper. Finally, apex return map is defined as a mapping from the current apex states, $b(n)$, to next apex states, $b(n+1)$, by using the chosen control inputs, $u$, as illustrated in Fig. 3(b).

\section{B. Simple Approximate Stance Map by Geyer et al.}

In this section, we briefly review the approximation method proposed in [10]. The equations of motion for the

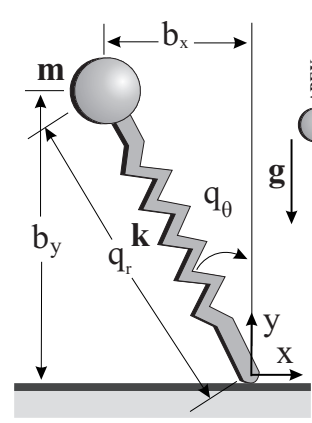

(a)

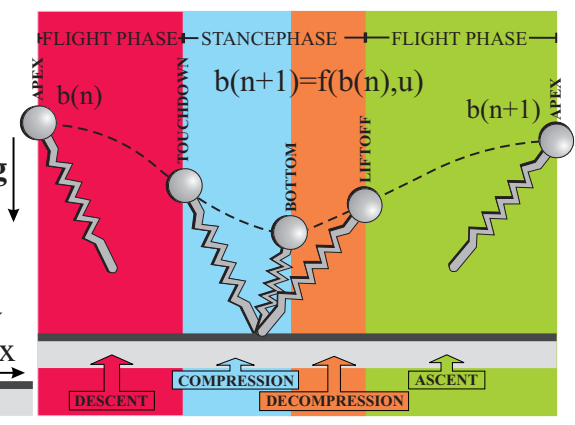

(b)
Fig. 3. The SLIP Model. (a) Coordinates and model parameters. (b) Locomotion phases (shaded regions) and transition events (boundaries).

TABLE I

NOTATION USED THROUGHOUT THE PAPER

\begin{tabular}{|c|l|}
\hline \multicolumn{2}{|c|}{ SLIP States } \\
\hline$q_{r}, q_{\theta}$ & Leg length and leg angle \\
$q_{\dot{r}}, q_{\dot{\theta}}$ & Leg compression and swing rates \\
$p_{r}, p_{\theta}$ & Radial and angular momenta \\
$q_{r_{t d}}, q_{\theta_{t d}}, t_{t d}$ & Touchdown leg length, angle and time \\
$q_{r_{b}}, q_{\theta_{b}}, t_{b}$ & Bottom leg length, angle and time \\
$q_{r_{l o}}, q_{\theta_{l o}}, t_{l o}$ & Liftoff leg length, angle and time \\
$b_{x}, b_{y}$ & Horizontal and vertical body positions \\
$b_{\dot{x}}, b_{\dot{y}}$ & Horizontal and vertical body velocities \\
$b_{y_{a}}, b_{\dot{x}_{a}}$ & Apex height and velocity \\
\hline \multicolumn{2}{|c|}{ SLIP Parameters } \\
\hline$m, g$ & Body mass and gravitational acceleration \\
$l_{0}, k$ & Leg rest length and leg stiffness \\
$E$ & Total mechanical energy \\
\hline
\end{tabular}

stance phase of SLIP in polar coordinates are given by

$$
\begin{aligned}
m \ddot{q}_{r} & =m q_{r} \dot{q}_{\theta}^{2}+k\left(l_{0}-q_{r}\right)-m g \cos \left(q_{\theta}\right), \\
0 & =\frac{d}{d t}\left(m q_{r}^{2} \dot{q}_{\theta}\right)+m g q_{r} \sin q_{\theta} .
\end{aligned}
$$

If a sufficiently small angular span $\Delta q_{\theta}$ is assumed for the stance phase, the effect of gravity can be linearized, yielding simplified equations of motion

$$
\begin{aligned}
m \ddot{q}_{r} & =m q_{r} \dot{q}_{\theta}^{2}+k\left(l_{0}-q_{r}\right)-m g_{s}, \\
\frac{d}{d t}\left(m q_{r}{ }^{2} \dot{q}_{\theta}\right) & =0,
\end{aligned}
$$

which are now integrable since the angular momentum, $p_{\theta}:=$ $m q_{r}{ }^{2} \dot{q}_{\theta}$ and the total energy

$$
E:=\frac{m}{2}{\dot{q_{r}}}^{2}+\frac{p_{\theta}{ }^{2}}{2 m q_{r}^{2}}+\frac{k}{2}\left(l_{0}-q_{r}\right)^{2}+m g_{s} q_{r}
$$

become constants of the motion. Defining the parameters

$$
\rho=\frac{q_{r}-l_{0}}{l_{0}} \leq 0, \epsilon=\frac{2 E}{m l_{0}^{2}}, \omega=\frac{p_{\theta}}{m l_{0}^{2}} \text { and } \omega_{0}=\sqrt{\frac{k}{m}},
$$

and substituting them into (1), yields

$$
\epsilon=\dot{\rho}^{2}+\omega^{2} /(1+\rho)^{2}+\omega_{0}{ }^{2} \rho^{2}+2 g(1+\rho) / l_{0} .
$$

If we also assume that the relative spring compression $|\rho| \ll 1$, then the term $1 /(1+\rho)^{2}$ can be approximated by a Taylor series expansion around zero to yield

$$
1 /\left.(1+\rho)^{2}\right|_{\rho=0}=1-2 \rho+3 \rho^{2}-O\left(\rho^{3}\right) .
$$


Under these assumptions and further simplifications [10], an analytical solution to $q_{r}(t)$ can be found as

$$
q_{r}(t)=l_{0}\left(1+a+b \sin \left(\hat{\omega}_{0} t\right)\right)
$$

where we define

$$
\begin{aligned}
\hat{\omega}_{0} & :=\sqrt{\omega_{0}^{2}+3 \omega^{2}}, \\
a & :=\left(\omega^{2}-g / l_{0}\right) / \hat{\omega}_{0}^{2}, \\
b & :=\sqrt{a^{2}+\left(\epsilon-\omega^{2}-2 g / l_{0}\right) / \hat{\omega}_{0}^{2}} .
\end{aligned}
$$

The equation (3) can also be used to determine the times for critical events relative to an unknown time origin as

$$
\begin{aligned}
t_{t d} & =(\pi-\arcsin (-a / b)) / \hat{\omega}_{0}, \\
t_{b} & =3 \pi / 2 \hat{\omega}_{0}, \\
t_{l o} & =(2 \pi+\arcsin (-a / b)) / \hat{\omega}_{0},
\end{aligned}
$$

assuming that $q_{r l o}=q_{r t d}=l_{0}$.

Given the analytical solution for the radial motion and the conservation of the angular momentum, we also have

$$
q_{\dot{\theta}}=\omega /(1+\rho)^{2}=\omega(1-2 \rho) .
$$

Recalling that $\left.\rho:=\left(q_{r}-l_{0}\right) / l_{0}=a+b \sin \left(\hat{\omega}_{0} t\right)\right)$, an analytic solution to the angular motion can be found as

$$
\begin{aligned}
q_{\theta}(t)= & q_{\theta t d}+\omega(1-2 a)\left(t-t_{t d}\right) \\
& +\frac{2 b \omega}{\hat{\omega}_{0}}\left[\cos \left(\hat{\omega}_{0} t\right)-\cos \left(\hat{\omega}_{0} t_{t d}\right)\right],
\end{aligned}
$$

where $t_{t d} \leq t \leq t_{l o}$, and $t_{t d}$ and $t_{l o}$ as in (4) and (6).

\section{Iterative Approximate Stance Map by Schwind et al.}

In [17], Schwind and Koditschek propose an analytic approximation to the nonintegrable stance dynamics of SLIP through an iterative application of the mean value theorem for integral operators. They showed that this iterative method converges to true SLIP dynamics under certain assumptions, which are unfortunately easily violated for non-symmetric trajectories. Furthermore, the presented form of their approximation only gives a map from bottom to apex and does not readily support the entire apex return map. In this section we briefly describe and extend on their method to enable comparisons with our proposed approximations.

The Hamiltonian function for the stance phase is given by

$H\left(q_{r}, p_{r}, q_{\theta}, p_{\theta}\right)=\frac{1}{2 m}\left(p_{r}^{2}+\frac{p_{\theta}^{2}}{q_{r}^{2}}\right)+\frac{1}{2} k\left(l_{0}-q_{r}\right)^{2}+m g q_{r} \cos \left(q_{\theta}\right)$.

Solving the equation $H\left(q_{r}, p_{r}, q_{\theta}, p_{\theta}\right)=E$ for $p_{r}$ yields

$$
\begin{aligned}
p_{r}=H^{\dagger}\left(q_{r}, q_{\theta}, p_{\theta}, E\right):= & \\
& \sqrt{2 m\left(E-\frac{1}{2} k\left(l_{0}-q_{r}\right)^{2}-m g q_{r} \cos \left(q_{\theta}\right)\right)-\frac{p_{\theta}^{2}}{q_{r}^{2}}},
\end{aligned}
$$

The approximation introduced in [17] gives the $n^{\text {th }}$ iteration for the bottom to liftoff map as

$$
\begin{aligned}
& \hat{t}_{s_{(n+1)}}\left(q_{r}\right)=t_{b}+m\left(q_{r}-q_{r_{b}}\right) / H_{n}^{\dagger}, \\
& \hat{q}_{\theta_{(n+1)}}\left(q_{r}\right)=q_{\theta_{b}}+\hat{p}_{\theta_{n}}\left(\hat{\xi}_{r}\right)\left(q_{r}-q_{r_{b}}\right) /\left(\hat{\xi}_{r}^{2} H_{n}^{\dagger}\right), \\
& \hat{p}_{\theta_{(n+1)}}\left(q_{r}\right)=p_{\theta_{b}}+m^{2} g \hat{\xi}_{r} \sin \left(\hat{q}_{\theta_{n}}\left(\hat{\xi}_{r}\right)\right)\left(q_{r}-q_{r_{b}}\right) / H_{n}^{\dagger}, \\
& \hat{p}_{r_{(n+1)}}\left(q_{r}\right)=H_{n+1}^{\dagger},
\end{aligned}
$$

such that the zeroth (initial) iteration can be any approximate analytical solution and $\hat{\xi}_{r}=3 q_{r_{t d}} / 4+q_{r} / 4$ and $H_{k}^{\dagger}:=$ $H^{\dagger}\left(\hat{\xi}_{r}, \hat{q}_{\theta_{k}}\left(\hat{\xi}_{r}\right), \hat{p}_{\theta_{k}}\left(\hat{\xi}_{r}\right), E\right)$.

A touchdown to bottom map can be similarly derived as

$$
\begin{aligned}
& \hat{t}_{s_{(n+1)}}\left(q_{r}\right)=t_{t d}-m\left(q_{r}-q_{r_{t d}}\right) / H_{n}^{\dagger} \\
& \hat{q}_{\theta_{(n+1)}}\left(q_{r}\right)=q_{\theta_{t d}}-\hat{p}_{\theta_{n}}\left(\hat{\xi}_{r}\right)\left(q_{r}-q_{r_{t d}}\right) /\left(\hat{\xi}_{r}^{2} H_{n}^{\dagger}\right) \\
& \hat{p}_{\theta_{(n+1)}}\left(q_{r}\right)=p_{\theta_{t d}}-m^{2} g \hat{\xi}_{r} \sin \left(\hat{q}_{\theta_{n}}\left(\hat{\xi}_{r}\right)\right)\left(q_{r}-q_{r_{t d}}\right) / H_{n}^{\dagger} \\
& \hat{p}_{r_{(n+1)}}\left(q_{r}\right)=-H_{n+1}^{\dagger} .
\end{aligned}
$$

The overall apex return map can then be obtained by combining the above approximations using the desired number of iterations with solutions to the flight dynamics. However, this formulation of the apex return map has one important unknown: the bottom leg length, $q_{r_{b}}$. Fortunately, even though an exact solution for the bottom leg length does not exist, several approximate solutions can be used. One such solution is given by the quartic equation arising from the total energy relation as

$$
\frac{k}{2} q_{r}^{4}+\left(m g-k l_{0}\right) q_{r}^{3}+\left(\frac{k l_{0}^{2}}{2}-E\right) q_{r}^{2}+\frac{p_{\theta}^{2}}{2 m}=0,
$$

for which a real analytic solution that is less than or equal to the rest leg length can be found. An alternative approximate solution is given by the approximate stance map of [10] as

$$
q_{r_{b}}=l_{0}(1+a-b),
$$

where $a$ and $b$ are as defined in Section II-B.

\section{APPROXIMATE STANCE MAP WITH GRAVITY CORRECTION}

In Section II, we reviewed two leading methods $[10,17]$ to find analytic approximations to the stance map of SLIP, both of which were based on assumptions of symmetric gaits. In this section, we extend the method presented in [10] with a gravity correction to yield a much larger domain of validity in the presence of non-symmetric trajectories.

As illustrated in Fig. 2, the angular momentum from touchdown to liftoff is conserved only for symmetric SLIP trajectories. Consequently, while the conservation of angular momentum is a reasonable assumption for symmetric steps, it becomes rather inaccurate for non-symmetric trajectories.

During stance (see Fig. 3), the effect of gravity on the angular momentum, $P(t)$, can be generally modeled as

$$
\begin{aligned}
P(t) & =P_{t_{0}}+\int_{t_{0}}^{t} \tau(\zeta) d \zeta \\
\tau(t) & :=m g q_{r}(t) \sin q_{\theta}(t),
\end{aligned}
$$

where $\tau(t)$ is the torque applied by the gravitational acceleration around the toe point. Generally, even the analytic approximations (3) and (8) are too complex for symbolic integration. Consequently, our method involves an $n$-point approximation to this integral, yielding

$$
P(t) \approx P_{t_{0}}+\left(t-t_{0}\right)\left(\frac{1}{n} \sum_{k=1}^{n} m g q_{r}[n] \sin q_{\theta}[n]\right) .
$$


We propose to model the total effect of gravity on the angular momentum from any initial to any final state during stance with a constant total correction, computed by using an approximation to the average leg length $q_{r_{a v}}\left(t_{i}, t_{f}\right)$ during this period. We then use this general formulation to compute a correction for the apex return map, using touchdown and liftoff as the initial and final states, respectively.

Using (3) and letting $t_{i}$ and $t_{f}$ be the initial and final state times such that $t_{t d} \leq t_{i}<t_{f} \leq t_{l o}$, we have

$$
\begin{aligned}
& q_{r_{a v}}\left(t_{i}, t_{f}\right) \approx \frac{1}{t_{f}-t_{i}} \int_{t_{i}}^{t_{f}} l_{0}\left(1+a+b \sin \left(\hat{\omega}_{0} t\right)\right) d t, \\
& =l_{0}(1+a)-\frac{b}{\hat{\omega}_{0}\left(t_{f}-t_{i}\right)}\left(\cos \left(\hat{\omega}_{0} t_{f}\right)-\cos \left(\hat{\omega}_{0} t_{i}\right)\right),
\end{aligned}
$$

where $a, b$ and $\hat{\omega}_{0}$ can be calculated by using related formulas in Section II-B. Using (9), the total effect of gravity becomes

$$
P_{c}:=\frac{\left(t_{f}-t_{i}\right) m g_{s} q_{r_{a v}}\left(t_{i}, t_{f}\right)}{2}\left(\sin q_{\theta}\left(t_{i}\right)+\sin q_{\theta}\left(t_{f}\right)\right),
$$

where $q_{\theta}(t)$, is as given in Section II-B.

We propose to incorporate $P_{c}$ into the approximation as a simple correction term added to the original angular momentum, $p_{\theta}$, otherwise assumed to be constant for the formulations in [10]. This yields a new angular momentum term

$$
\hat{p}_{\theta}=p_{\theta}+P_{c} .
$$

which replaces $p_{\theta}$ in all derivations. Using touchdown and liftoff times as the initial and final states yields our corrective method for the apex return map.

\section{PERFORMANCE ANALYSIS}

\section{A. Simulation Environment and Performance Criteria}

Our interest in analytic approximations to the stance dynamics of SLIP arises from our need to compute the apex return map for a given set of controls.(i.e. touchdown angle and leg stiffness). Therefore, the most important performance criteria for us is the accuracy of the predicted apex position and velocity. To this end, we use two measures to quantify the prediction performance of both our method and existing methods: Normalized percentage errors in the apex position and liftoff velocity predictions are respectively defined as

$$
\begin{aligned}
P E_{a p} & =100 \frac{\left\|\left(b_{x_{a}}, b_{y_{a}}\right)-\left(\hat{b}_{x_{a}}, \hat{b}_{y_{a}}\right)\right\|_{2}}{\left\|\left(b_{x_{a}}, b_{y_{a}}\right)\right\|_{2}}, \\
P E_{l o v} & =100 \frac{\left\|\left(b_{\dot{x}_{l o}}, b_{\dot{y}_{l o}}\right)-\left(\hat{b}_{\dot{x}_{l o}}, \hat{b}_{\dot{y}_{l o}}\right)\right\|_{2}}{\left\|\left(b_{\dot{x}_{l o}}, b_{\dot{y}_{l o}}\right)\right\|_{2}} .
\end{aligned}
$$

We use the liftoff velocity rather than the apex velocity to ensure that normalization is practical even for non-symmetric gaits for which the apex velocity may become zero.

In order to compare different methods of approximation, we ran simulations spanning four different dimensions of initial states and control inputs: the apex height $\left(b_{y_{a}}\right)$, the apex velocity $\left(b_{\dot{x}_{a}}\right)$, the spring constant $(k)$ and the relative touchdown angle $q_{\theta_{t d_{r e l}}}:=q_{\theta_{t d}}-q_{\theta_{t d_{n}}}{ }^{1}$. The ranges

\footnotetext{
${ }^{1} q_{\theta_{t d_{n}}}$ is the neutral touchdown angle which results in a symmetric SLIP trajectory and depends on the initial conditions. For each simulation, we numerically calculated this angle to be used as the origin for our plots.
}

considered for these dimensions were determined based on biomechanics literature as well as structural properties of various legged robots. In particular, experiments on humans (with $80 \mathrm{~kg}$ mass and $1 \mathrm{~m}$ leg length on average) running at different speeds (in the range $2.5-6.5 \mathrm{~m} / \mathrm{s}$ ) reveals that their leg stiffnesses are in the range $[10,50] \mathrm{kN} / \mathrm{m}[4]$. In the robotic domain, the small hexapod robot RHex [15], has an approximate mass of $10 \mathrm{~kg}$, leg length of $0.25 \mathrm{~m}$ and compliant legs with stiffness of around $2000 \mathrm{~N} / \mathrm{m}$ for each leg. Based on a dimensionless stiffness measure $\hat{k}=\frac{l_{0}}{m g} k$, these examples motivate our choice of simulation parameters of Table II and control input ranges of $k \in[125,1000] \mathrm{N} / \mathrm{m}$ and $q_{\theta_{t d_{r e l}}} \in[-0.4,0.4] \mathrm{rad}$.

TABLE II

SiMULATION PARAMETERS

\begin{tabular}{|c|c|c|c|c|}
\hline $\mathrm{m}(\mathrm{kg})$ & $l_{0}(\mathrm{~m})$ & $\mathrm{g}\left(\mathrm{m}^{2} / \mathrm{s}\right)$ & $b_{y_{a}}(\mathrm{~m})$ & $b_{\dot{x}_{a}}(\mathrm{~m} / \mathrm{s})$ \\
\hline \hline 1 & 1 & 9.81 & $1.1-1.5$ & $0-8$ \\
\hline
\end{tabular}

For each of our simulations, we checked whether they satisfied two conditions to ensure that we can support meaningful comparisons of all approximations to the stance map. Firstly, due to the hybrid nature of SLIP locomotion, certain stance trajectories never leave the ground or prevent foot protraction. Consequently, we restricted our domains to exclude simulations where the next apex height is smaller than the rest length of the leg spring, ensuring that there are no limitations on the touchdown angle for the next step. Second, in order to preserve similarity to results presented in both [17] and [10], we restricted the maximum allowable leg compression to a maximum of $25 \%$ and excluded trajectories that violate this condition. From among a total of 25500 initial states and control inputs, 10264 were found to satisfy these two conditions.

We computed "ground truth" through numerical integration of SLIP dynamics within MATLAB using a variable time-step, fourth order Runge-Kutta integrator. We then computed approximate estimates of the apex states based on two previously proposed approximations and our new gravity correction scheme and compared estimation performances uwing the error criteria defined above. For the Schwind approximations, we used the 10th iterate (after which further iterations yield no improvements) to make sure we obtained the best possible performance for their method.

\section{B. Simulation Results}

Our simulation results are illustrated in Fig. 4, where we show the mean, standard deviation and maximum values for the apex position and liftoff velocity percentage errors, $P E_{a p}$ and $P E_{l o v}$, across all valid simulations and all three approximation methods. Our results show that there is a notable performance improvement on the average for our proposed gravity correction method compared both to Geyer's and Schwind's approximations.

A more informative comparison between different approximation alternatives can be achieved by investigating the estimation performance as a function of the relative touchdown angle. Since our method is expected to perform 


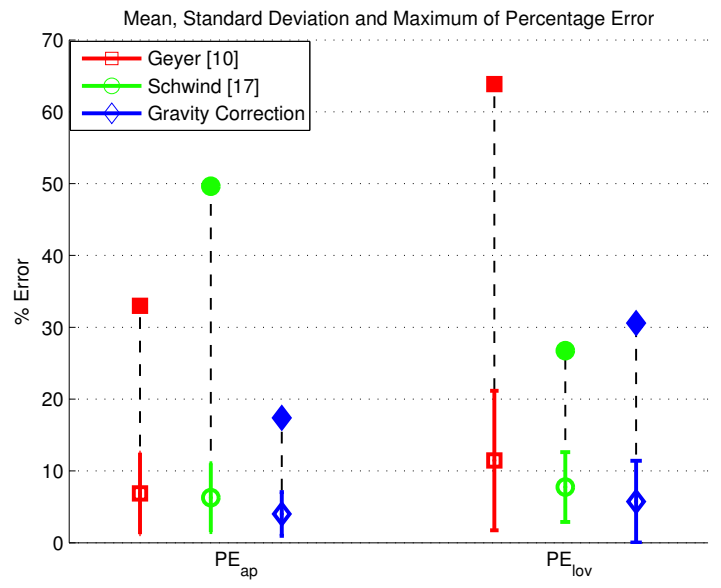

Fig. 4. Approximation performances for the stance maps of Geyer [10], Schwind [17] and our proposed Gravity Correction method. $P E_{a p}$ (left) and $P E_{l o v}$ (right) are apex position and liftoff velocity percentage errors. Empty markers, filled markers and colored vertical bars represent mean, maximum and standard deviations of associated approximations.

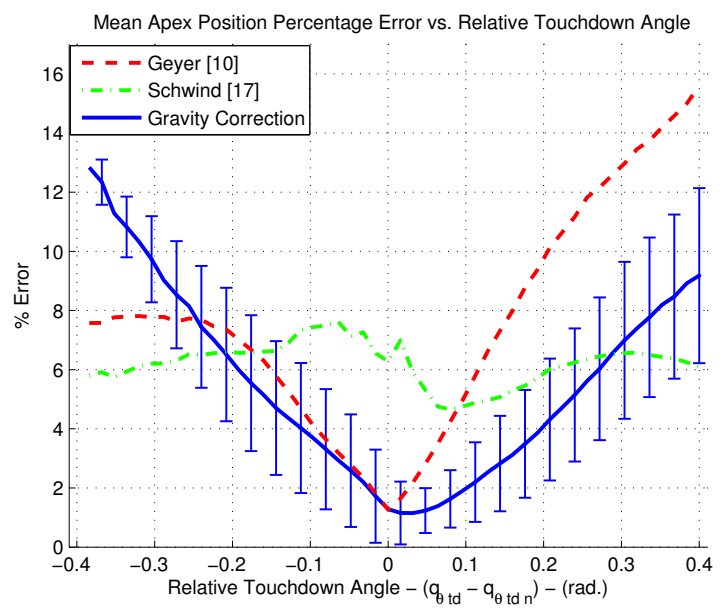

Fig. 5. Mean Apex Position Percentage Error $\left(P E_{a p}\right)$ versus Relative Touchdown Angle $\left(q_{\theta_{t d}}-q_{\theta_{t d_{n}}}\right)$. The vertical bars represent standard deviations for the approximate stance map with gravity correction.

well for non-symmetric trajectories, its error performance should be better than the alternatives for nonzero relative angle values. As illustrated by Figures 5 and 6 , this was indeed the case both error measures, $P E_{a p}$ and $P E_{l o v}$.

In almost all cases, our approximation performed better than Geyer's method for non-symmetric trajectories. Note that these two approximations are expected to perform identically for symmetric gaits (zero relative touchdown angle), which is also confirmed by their almost identical estimation performance for $q_{\theta_{t d_{\text {rel }}}} \in[-0.1,0] \mathrm{rad}$.

On the other hand, Schwind's iterative approximation has an almost uniform performance profile, relatively independent of symmetry. Consequently, it performs better than our approximation for some non-symmetric trajectory regions far out into the touchdown angle spectrum. However, these regions correspond to rather extreme transient conditions unlikely to be observed for locomotion on reasonable terrain. Furthermore, some of their method's performance can be attributed to the fact that we used the 10th iterate of their

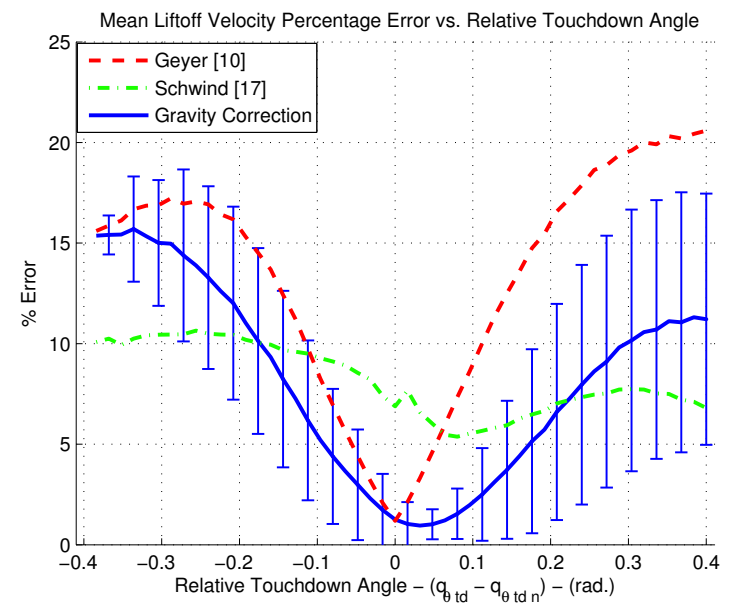

Fig. 6. Mean Liftoff Velocity Percentage Error $\left(P E_{l o v}\right)$ versus Relative Touchdown Angle $\left(q_{\theta_{t d}}-q_{\theta_{t d_{n}}}\right)$. The vertical bars represent the standard deviations for the approximate stance map with gravity correction.

approximations rather than more reasonable ones such as the first or second iterate for which the analytical nature of the approximations can still be preserved.

Overall, our gravity correction scheme performs best for relative touchdown angles in the range of $\left[\begin{array}{ll}-0.2 & 0.2\end{array}\right] \mathrm{rad}$. Fortunately, angles outside this range correspond to very sudden changes in the locomotion and can safely be left unused by a reasonable planner cognizant of the limitations of available approximations.

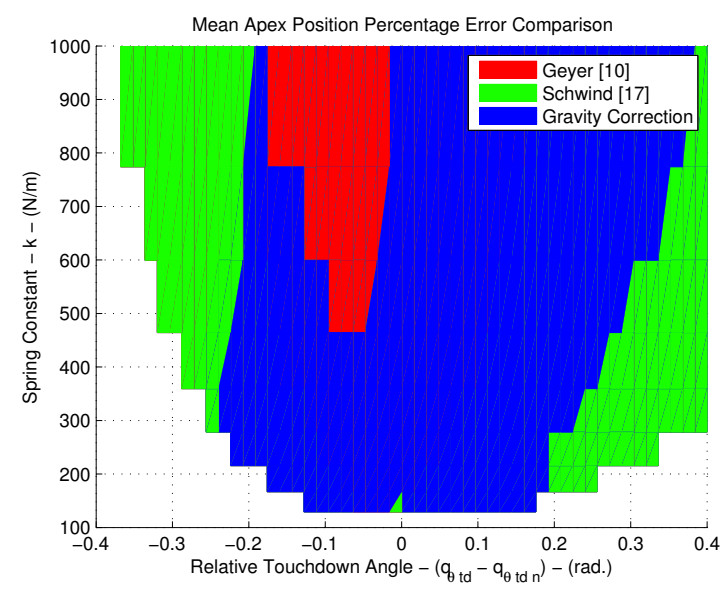

Fig. 7. Comparison of the mean Apex Position Percentage Errors. Colored regions show where the associated approximation performs better.

Finally, Figs. 7 and 8 illustrate regions in the control input space for which different approximants produce the best error performance. Schwind's method once again, is observed to have good performance far from symmetric gaits. When the relative touchdown angle is in the range $[-0.2,0.2] \mathrm{rad}$, our gravity correction scheme has the best performance for both error measures. In Fig. 7, even though Geyer's approximations seem to be better than ours for larger leg stiffnesses and nearly symmetric gaits, their performance is actually almost identical to ours in those areas as can be observed from corresponding regions in Fig. 5.

As illustrated by these results, our proposed method signif- 


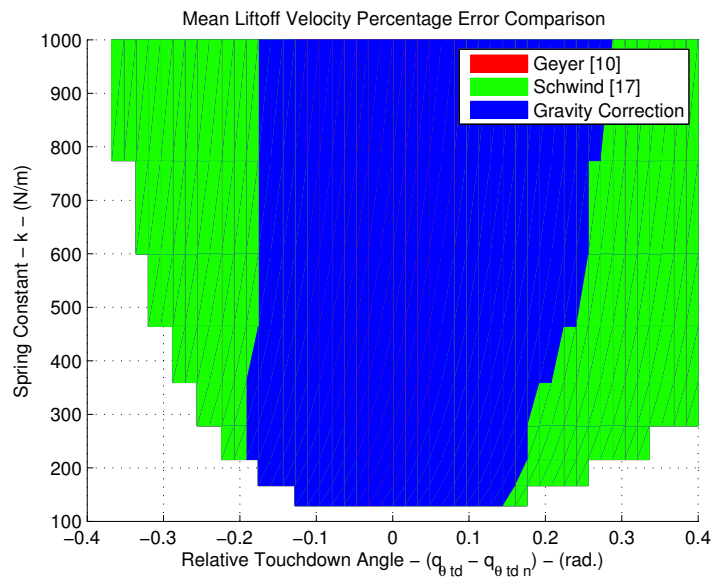

Fig. 8. Comparison of the mean Liftoff Velocity Percentage Errors. Colored regions show where the associated approximation performs better.

icantly improves the accuracy of approximations presented in [10]. When compared to Schwind's iterative approximations presented in [17], our approximation also performs better for the most commonly used subset of non-symmetric trajectories. It is important to note that the higher iterates of the Schwind approximations have much more complicated analytical forms, a significant handicap for the design of control algorithms and dynamic locomotion planning for SLIP. Our approximations, even with the gravity correction, have a very simple analytical form that can easily support control algorithms for dynamical locomotion and footstep planning for the SLIP model.

\section{CONCLUSIONS AND FUTURE WORK}

In this paper, we proposed a novel gravity correction method to improve the performance of the previously proposed analytic approximations to the stance dynamics of the SLIP model in [10]. Our method is based on the hypothesis that for non-symmetric locomotion trajectories, the effect of gravity on the total angular momentum can be summarized with a constant correction term. We demonstrated that this is indeed the case through comparisons of estimation errors for both Geyer's method, our method as well as a different analytic approximation method proposed in [17]. Our method was found to perform best for relative touchdown angles in the range [- 0.20 .2$]$ rad. For gaits that are sufficiently far from symmetric, Schwind's iterative stance map was observed to have better performance but under the condition that it is iterated until convergence, which usually results in unacceptable analytical complexity. Overall, our method seems to present the best combination of accuracy and simplicity for non-symmetric SLIP trajectories and is suitable for the design of footstep planning algorithms that rely on the use of transient stepping behavior.

Even though it was left outside the scope of the present paper, our approximations can also be easily applied to using tunable stiffness during stance as a control input as introduced in [14] and also embodied in the passive dynamics of the BiMASC leg [13]. This aspect turns out to be critical for nontrivial planning tasks with the SLIP model since it allows inducing changes in the total energy of the system, allowing finer control over possible maneuvers.

Our longer term goal is the design of reactive planning controllers for the SLIP model, which can in turn be applied to more complex legged robots through passive or active embedding of the SLIP model. To this end, we believe that analytic approximations to the dynamic behavior of this model will be invaluable both in the design of controllers that can accurately and efficiently regulate its discrete control inputs as well as in the analytical characterization of such controllers for planning purposes. Our proposed method fills a gap in this area and provides an analytic approximation that remains valid for a large range of control inputs and initial conditions of the SLIP model.

\section{REFERENCES}

[1] R. M. Alexander. Three uses for springs in legged locomotion. International Journal of Robotics Research, 9(2):53-61, 1990.

[2] R. M. Alexander and A. S. Jayes. Vertical movement in walking and running. Journal of Zoology, London, 185:27-40, 1978.

[3] R. Altendorfer, U. Saranli, H. Komsuoglu, D. Koditschek, H. B. Brown, M. Buehler, N. Moore, D. McMordie, and R. Full. Evidence for Spring Loaded Inverted Pendulum Running in a Hexapod Robot. In Proceedings of the International Symposium on Experimental Robotics, Honolulu, HI, 2001.

[4] A. Arampatzis, G.-P. Briggemann, and V. Metzler. The effect of speed on leg stiffness and joint kinematics in human running. Journal of Biomechanics, 32:1349-1353, 1999.

[5] R. Blickhan and R. J. Full. Similarity in multilegged locomotion: Bouncing like a monopode. Journal of Comparative Physiology A: Neuroethology, Sensory, Neural, and Behavioral Physiology, 173(5):509-517, November 1993.

[6] R. R. Burridge, A. A. Rizzi, and D. E. Koditschek. Sequential composition of dynamically dexterous robot behaviors. International Journal of Robotics Research, 18(6):534-555, 1999.

[7] S. G. Carver. Control of a spring-mass hopper. $\mathrm{PhD}$ thesis, Cornell University, Ithaca, NY, USA, 2003.

[8] C. T. Farley and D. P. Ferris. Biomecahnics of Walking and Running: Center of Mass Movements to Muscle Action. Excercise and Sport Science Reviews, 26:253-283, 1998.

[9] R. J. Full and D. E. Koditschek. Templates and Anchors: Neuromechanical Hypotheses of Legged Locomotion. Journal of Experimental Biology, 202:3325-3332, 1999.

[10] H. Geyer, A. Seyfarth, and R. Blickhan. Spring-mass running: simple approximate solution and application to gait stability. Journal of Theoretical Biology, 232:315-328, February 2005.

[11] P. Gregorio, M. Ahmadi, and M. Buehler. Design, Control, and Energetics of an Electrically Actuated Legged Robot. Transactions on Systems, Man, and Cybernetics, 27(4):626-634, August 1997.

[12] P. Holmes. Poincaré, celestial mechanics, dynamical-systems theory and "chaos". Physics Reports (Review Section of Physics Letters), 193:137-163, September 1990.

[13] J. W. Hurst, J. Chestnutt, and A. Rizzi. Design and Philosophy of the BiMASC, a Highly Dynamic Biped. In Proc. of the Int. Conf. on Robotics and Automation, April 2007.

[14] M. H. Raibert. Legged robots that balance. MIT Press, Cambridge, MA, USA, 1986.

[15] U. Saranli and D. E. Koditschek. Template based control of hexapedal running. In Proc. of the Int. Conf. On Robotics and Automation, volume 1, pages 1374-1379, Taipei, Taiwan, September 2003.

[16] W. J. Schwind. Spring loaded inverted pendulum running: a plant model. PhD thesis, University of Michigan, Ann Arbor, MI, USA, 1998.

[17] W. J. Schwind and D. E. Koditschek. Approximating the Stance Map of a 2 DOF Monoped Runner. Journal of Nonlinear Science, 10(5):533-588, 2000.

[18] G. Zeglin. The Bow Leg Hopping Robot. PhD thesis, Carnegie Mellon University, Pittsburgh, PA, USA, October 1999. 